\title{
MAURICE GAULTIER
}

\section{MiKel LEZAUN}

\section{Spectral study of a self-adjoint operator on $L^{2}(\Omega)$ related with a Poincaré type constant}

Annales de la faculté des sciences de Toulouse $\sigma^{e}$ série, tome 5, no 1 (1996), p. 105-123

<http://www.numdam.org/item?id=AFST_1996_6_5_1_105_0>

(C) Université Paul Sabatier, 1996, tous droits réservés.

L'accès aux archives de la revue "Annales de la faculté des sciences de Toulouse » (http://picard.ups-tlse.fr/ annales/) implique l'accord avec les conditions générales d'utilisation (http://www.numdam.org/conditions). Toute utilisation commerciale ou impression systématique est constitutive d'une infraction pénale. Toute copie ou impression de ce fichier doit contenir la présente mention de copyright.

\section{Numdam}

Article numérisé dans le cadre du programme

Numérisation de documents anciens mathématiques

http://www.numdam.org/ 


\title{
Spectral study of a self-adjoint operator on $L^{2}(\Omega)$ related with a Poincaré type constant ${ }^{(*)}$
}

\author{
Maurice GaultieR $^{(1)}$ and Mikel Lezaun ${ }^{(2)}$
}

RÉsuMÉ. - Soit $\Omega$ un ouvert borné et connexe de $\mathbb{R}^{N}, N \geq 2$, de frontière lipschitzienne. L'espace $H_{0}^{1}(\Omega)$ est muni de la norme du gradient. L'inégalité suivante a lieu pour les éléments de $L^{2}(\Omega)$.

$$
|u|_{L^{2}(\Omega)}^{2}-\frac{1}{\operatorname{mes}(\Omega)}\left|\int_{\Omega} u(x) \mathrm{d} x\right|^{2} \leq C(\Omega) \sum_{i=1}^{N}\left\|\frac{\partial u}{\partial x_{i}}\right\|_{H^{-1}(\Omega)}^{2}
$$

où $C(\Omega)>0$ ne dépend que de $\Omega$. À l'aide d'un opérateur autoadjoint sur $L^{2}(\Omega)$, on caractérise la meilleure constante dans l'inégalité précédente. Lorsque $\Omega$ est une boule de $\mathbb{R}^{N}, N \geq 2$, on fait l'analyse spectrale de cet opérateur et on montre que la meilleure valeur de la constante est $N$.

Abstract. - Let $\Omega$ be a connected bounded open set in $\mathbb{R}^{N}, N \geq 2$, with lipschitzian boundary. $H_{0}^{1}(\Omega)$ is equipped with the gradient norm. The following inequality holds for the elements of $L^{2}(\Omega)$ :

$$
|u|_{L^{2}(\Omega)}^{2}-\frac{1}{\operatorname{mes}(\Omega)}\left|\int_{\Omega} u(x) \mathrm{d} x\right|^{2} \leq C(\Omega) \sum_{i=1}^{N}\left\|\frac{\partial u}{\partial x_{i}}\right\|_{H^{-1}(\Omega)}^{2}
$$

where $C(\Omega)>0$ depends only on $\Omega$. This paper provides a characterization of the best constant in the previous inequality using a self-adjoint operator on $L^{2}(\Omega)$. When $\Omega$ is a ball in $\mathbb{R}^{N}, N \geq 2$, the spectral study of this operator is made and in this case, we obtain that the best constant is $N$.

(*) Reçu le 12 janvier 1994

(1) Université de Bordeaux I et C.R.M. B, 351 cours de la Libération, F-33405 Talence Cedex (France)

(2) Departemento de Matemática Aplicada, Estadística e Investigagación Operativa, Facultad de Ciencias, Universidad del País Vasco, Apartado 644, Bilbao (Spain) 


\section{Introduction}

Throughout this paper $\Omega$ is a connected bounded open set in $\mathbb{R}^{N}, N \geq 2$, and its boundary $\Gamma$ is Lipschitz-continuous as [5]. The space $H_{0}^{1}(\Omega)$ will always be equipped with the gradient norm. Derivates of functions on $\Omega$ will be taken in the sense of distributions.

We denote by $H^{-1}(\Omega)$ the dual space of $H_{0}^{1}(\Omega)$ normed by:

$$
\|f\|_{H^{-1}(\Omega)}=\sup _{\substack{v \in H_{0}^{1}(\Omega) \\ v \neq 0}}\left(\frac{\langle f, v\rangle}{\|v\|_{H_{0}^{1}(\Omega)}}\right)
$$

where $\langle\cdot, \cdot\rangle$ denotes the duality between $H^{-1}(\Omega)$ and $H_{0}^{1}(\Omega)$.

The following inequality

$$
|u|_{L^{2}(\Omega)}^{2} \leq C(\Omega) \sum_{i=1}^{N}\left\|\frac{\partial u}{\partial x_{i}}\right\|_{H^{-1}(\Omega)}^{2}, \quad \forall u \in L^{2}(\Omega), \int_{\Omega} u(x) \mathrm{d} x=0
$$

or the equivalent inequality: $\forall u \in L^{2}(\Omega)$,

$$
|u|_{L^{2}(\Omega)}^{2}-\frac{1}{\operatorname{mes}(\Omega)}\left|\int_{\Omega} u(x) \mathrm{d} x\right|^{2} \leq C(\Omega) \sum_{i=1}^{N}\left\|\frac{\partial u}{\partial x_{i}}\right\|_{H^{-1}(\Omega)}^{2}
$$

where $C(\Omega)>0$ depends only on $\Omega$ occurs in very many problems in the mechanics of continuous media [4]. Constant $C(\Omega)$ then occurs in the conditions for the uniqueness and sometimes for the existence of solutions. Knowledge of a value of $C(\Omega)$ is also important for the Numerical Analysis of these problems.

This paper provides a characterization of the best constant $P(\Omega)$ in inequalities (1)-(2) using a self-adjoint operator on $L^{2}(\Omega)$. Except if $\Omega$ is a ball in $\mathbb{R}^{N}$, the explicit value of this best constant $P(\Omega)$ is out of reach. In the particular case where $\Omega$ is a rectangle in $2-\mathrm{D}$, we obtain an approximate value of this best constant $P(\Omega)$. 
The reader will recall that if $u \in H^{1}(\Omega)$, we have the following inequality, called Poincaré's inequality:

$$
|u|_{L^{2}(\Omega)}^{2}-\frac{1}{\operatorname{mes}(\Omega)}\left|\int_{\Omega} u(x) \mathrm{d} x\right|^{2} \leq K(\Omega) \sum_{i=1}^{N}\left|\frac{\partial u}{\partial x_{i}}\right|_{L^{2}(\Omega)}^{2},
$$

where $K(\Omega)>0$ depends only on $\Omega$. It is well known ([3]) that the best constant in this inequality, called Poincaré's constant, is the inverse of the smallest (positive) eigenvalue of the operator $-\Delta$ (in $L^{2}(\Omega)$ ) for the Neumann problem. The explicit value of this constant is in general out of reach.

This paper is organized as follows.

Section 2 introduces some function spaces and we draw attention to an important inequality due to J. Necas [6] for the elements of $L^{2}(\Omega)$ which plays an essential role in the proof of inequalities (1)-(2).

In Section 3, we propose a proof of coercivity inequality (2) and, in particular, as a consequence, we obtain the following well known result:

$$
\text { if } f \in D^{\prime}(\Omega) \text { with } \operatorname{grad}(f) \in\left(H^{-1}(\Omega)\right)^{N}, \quad \text { then } f \in L^{2}(\Omega) \text {. }
$$

In Section 4, we prove that the best constant in inequalities (1)-(2) is the inverse of the smallest spectral value of the operator $T=-\operatorname{div} \circ(-\Delta)^{-1}$ 。 $\operatorname{grad}$ on $L^{2}(\Omega) / \mathbb{R}$.

In Section 5, we prove that the inverse of this best constant $P(\Omega)$ is the limit of a decreasing positive sequence which has for general term the smallest eigenvalue of the matrix corresponding to a positive definite quadratic form on a suitable finite dimensional euclidean space.

In Section 6, we consider two particular cases:

- $\Omega$ is a rectangle in 2-D. Using an appropriate basis of $L^{2}(\Omega)$, we obtain an approximate value of this constant $P(\Omega)$;

- $\Omega$ is a ball in $\mathbb{R}^{N}$ with $N \geq 2$. We make the complete spectral study of operator $-\operatorname{div} \circ(-\Delta)^{-1} \circ \operatorname{grad}$ and we obtain that $P(\Omega)=N$. 


\section{Preliminaries}

Throughout this paper we suppose for simplicity that all functions are real.

We use the usual product topology on the product spaces. We denote

$$
\partial_{i}=\frac{\partial}{\partial x_{i}} .
$$

For $u=\left(u_{1}, \ldots, u_{N}\right) \in\left(D^{\prime}(\Omega)\right)^{N}$, we set $\Delta u=\left(\Delta u_{1}, \ldots, \Delta u_{N}\right)$. For $f=D^{\prime}(\Omega)$, we set $\operatorname{grad}(f)=\left(\partial_{1} f, \ldots, \partial_{N} f\right)$.

In $L^{2}(\Omega)$ the Hilbert norm and the scalar product are written $|\cdot|_{2}$ and $(\cdot, \cdot)_{2}$.

Let $M(\Omega)$ be the closed subspace of $L^{2}(\Omega)$ of functions of zero mean (orthogonal to constants):

$$
M(\Omega)=\left\{u \in L^{2}(\Omega) \mid \int_{\Omega} u(x) \mathrm{d} x=0\right\},
$$

$M(\Omega)$ is equipped with the norm induced by Hilbert space $L^{2}(\Omega)$.

The quotient space $L^{2}(\Omega) / \mathbb{R}$, equipped with the usual quotient norm, is isometrically isomorphic to $M(\Omega)$. This isomorphism maps each equivalence class to its element of minimal norm, which is also the unique element of mean zero in the class. By convention we write $L^{2}(\Omega) / \mathbb{R} \equiv M(\Omega)$.

We recall that $H_{0}^{1}(\Omega)$ is equipped with the gradient norm, denoted by $\|\cdot\|$ and $H^{-1}(\Omega)$ is equipped with the dual norm. $\left(H_{0}^{1}(\Omega)\right)^{N}$ is isomorphic to $\left(H^{-1}(\Omega)\right)^{N}$ and $-\Delta$ is this isometric isomorphism.

For simplicity, in the remainder of this paper, we shall write indiscriminately $\|\cdot\|$ for the norm on $H_{0}^{1}(\Omega)$ or on $\left(H_{0}^{1}(\Omega)\right)^{N}$ and $\|\cdot\|_{-1}$ (resp. $((\cdot, \cdot))_{-1}$ ) for the norm (resp. scalar product) on $H^{-1}(\Omega)$ or on $\left(H^{-1}(\Omega)\right)^{N}$.

We introduce the following closed subspaces of $\left(H_{0}^{1}(\Omega)\right)^{N}$ :

$$
\begin{gathered}
V=\left\{u \in\left(H_{0}^{1}(\Omega)\right)^{N} \mid \operatorname{div}(u)=0\right\}, \\
V^{\perp}: \text { the subspace of }\left(H_{0}^{1}(\Omega)\right)^{N} \text { orthogonal to } V .
\end{gathered}
$$

$V$ and $V^{\perp}$ are equipped with the norm induced by $\left(H_{0}^{1}(\Omega)\right)^{N}$ (for properties of $V$ see eg. [7]). 
The important inequality which follows is proved in [6].

There exist a positive constant $N(\Omega)$ which depends only on $\Omega$ such that:

$$
|u|_{2} \leq N(\Omega)\left(\|u\|_{-1}+\|\operatorname{grad}(u)\|_{-1}\right), \quad \text { for all } u \in L^{2}(\Omega) .
$$

\section{Poincaré type inequality on $L^{2}(\Omega)$}

Proposition 1. - There exist a constant $C(\Omega) \geq 1$, depending only on $\Omega$, such that:

$$
|u|_{2}^{2} \leq C(\Omega)\|\operatorname{grad}(u)\|_{-1}^{2}+\frac{1}{\operatorname{mes}(\Omega)}\left(\int_{\Omega} u(x) \mathrm{d} x\right)^{2}, \quad \forall u \in L^{2}(\Omega) .
$$

Proof. - grad $\in L\left(L^{2}(\Omega),\left(H^{-1}(\Omega)\right)^{N}\right)$ and the kernel of grad is $\mathbb{R}$ because $\Omega$ is connected. Consequently, grad is a linear continuous injective mapping from $L^{2}(\Omega) / \mathbb{R}$ into $\left(H^{-1}(\Omega)\right)^{N}$.

Now we are going to show, by contradiction, that grad is bicontinuous.

We suppose that $\operatorname{grad}^{-1}$ is not bounded at 0 . Then there exists a sequence $\left\{\widehat{u}_{p}\right\}$ of $L^{2}(\Omega) / \mathbb{R}$ such that $\left|\widehat{u}_{p}\right|_{L^{2}(\Omega) / \mathbb{R}}=1$ and

$$
\lim _{p \rightarrow \infty}\left\|\operatorname{grad}\left(\widehat{u}_{p}\right)\right\|_{-1}=0
$$

whence $\left|u_{p}^{0}\right|_{2}=1$ and

$$
\lim _{p \rightarrow \infty}\left\|\operatorname{grad}\left(u_{p}^{0}\right)\right\|_{-1}=0
$$

where $u_{p}^{0}$ is the unique element of $\widehat{u}_{p}$ of minimal norm.

Taking into account that the injection from $L^{2}(\Omega)$ into $H^{-1}(\Omega)$ is compact, there exist a subsequence $\left\{u_{p_{k}}^{0}\right\}$ which converges in $H^{-1}(\Omega)$.

It follows from (3) that there exist $u^{0} \in L^{2}(\Omega)$ such that:

$$
\lim _{k \rightarrow \infty} u_{p_{k}}^{0}=u^{0} \text { in } L^{2}(\Omega) \text { and } \lim _{k \rightarrow \infty}\left\|\operatorname{grad}\left(u_{p_{k}}^{0}\right)\right\|_{-1}=0 .
$$


This result implies that $\operatorname{grad}\left(u^{0}\right)=0$, therefore

$$
\lim _{k \rightarrow \infty} \widehat{u}_{p_{k}}=\widehat{0}
$$

in contradiction with the definition of sequence $\left\{\widehat{u}_{p}\right\}$.

Consequently, there exist a positive constant $C(\Omega)$, depending only on $\Omega$, such that:

$$
|u|_{2}^{2} \leq C(\Omega)\|\operatorname{grad}(u)\|_{-1}^{2}, \quad \text { for all } u \in M(\Omega) \equiv L^{2}(\Omega) / \mathbb{R} .
$$

On the other hand, for each $u \in L^{2}(\Omega)$, there exist an unique element $v \in M(\Omega)$ such that

$$
u=v+\frac{1}{\operatorname{mes}(\Omega)} \int_{\Omega} u(x) \mathrm{d} x .
$$

Hence

$$
|u|_{2}^{2}=|v|_{2}^{2}+\frac{1}{\operatorname{mes}(\Omega)}\left(\int_{\Omega} u(x) \mathrm{d} x\right)^{2} .
$$

Then, it follows from inequality (4),

$$
|u|_{2}^{2} \leq C(\Omega)\|\operatorname{grad}(u)\|_{-1}^{2}+\frac{1}{\operatorname{mes}(\Omega)}\left(\int_{\Omega} u(x) \mathrm{d} x\right)^{2}, \quad \forall u \in L^{2}(\Omega) .
$$

As $|\operatorname{div}(\phi)|_{2} \leq\|\phi\|$ for all $\phi \in\left(H_{0}^{1}(\Omega)\right)^{N}[7$, p. 140],

$$
\|\operatorname{grad}(u)\|_{1}=\operatorname{Sup}_{\|v\| \leq 1}\langle\operatorname{grad}(u), v\rangle=\operatorname{Sup}_{\|v\| \leq 1}(u, \operatorname{div}(v))_{2} \leq|u|_{2}|\operatorname{div}(v)|_{2} .
$$

Therefore $\|\operatorname{grad}(u)\|_{-1} \leq|u|_{2}$. Then, it follows from (4) that $C(\Omega) \geq 1$.

Corollary 1. - $-\operatorname{div} \circ(-\Delta)^{-1} \circ \mathrm{grad}$ is an isomorphism from $M(\Omega)$ onto $M(\Omega)$.

Proof. - We identify $L^{2}(\Omega)$ with its dual. It follows from proposition 1 that grad is an isomorphism from $M(\Omega)$ into $\left(H^{-1}(\Omega)\right)^{N}$. Then its adjoint, - div, is an isomorphism from $\left(H_{0}^{1}(\Omega)\right)^{N} / V$ onto $M(\Omega)$. By transposition, grad is an isomorphism from $M(\Omega)$ onto the dual space of $\left(H_{0}^{1}(\Omega)\right)^{N} / V$ that is onto $V^{0}$ (the annihilator of $V$ ). It is not difficult to see that $V^{0}=-\Delta\left(V^{\perp}\right)$. 
We deduce from corollary 1 the following well known result.

Corollary 2. - If $f \in D^{\prime}(\Omega)$ and $\operatorname{grad}(f) \in\left(H^{-1}(\Omega)\right)^{N}$, then $u \in L^{2}(\Omega)$.

Proof. - Let $v \in(D(\Omega))^{N}$ such that $\operatorname{div}(v)=0$. Then we have:

$$
\langle\operatorname{grad}(f), v\rangle=-\langle f, \operatorname{div}(v)\rangle=0 .
$$

Therefore $\operatorname{grad}(f) \in V^{0}$. Thus, there exist $g \in L^{2}(\Omega) / \mathbb{R}$ such that $\operatorname{grad}(f)=\operatorname{grad}(g)$. It follows that $f=g+C$ because $\Omega$ is connected.

Notation. - In the remainder of this paper, the best value of constant $C(\Omega)$ in inequalities (4)-(5) is denoted by $P(\Omega)$ :

$$
P(\Omega)^{-1}=\operatorname{Inf}_{\substack{u \in M(\Omega) \\ u \neq 0}} \frac{\|\operatorname{grad}(u)\|_{-1}^{2}}{|u|_{2}^{2}} .
$$

4. The operator related with the Poincaré type constant $P(\Omega)$

From corollary 1 , the operator $T=-\operatorname{div} \circ(-\Delta)^{-1} \circ \operatorname{grad}$ is an isomorphism from $M(\Omega)$ onto $M(\Omega)$. Moreover, for all $u \in M(\Omega)$ :

$$
(T u, u)_{2}=\sum_{i=1}^{N}\left\langle\partial_{i} u,(-\Delta)^{-1} \circ \partial_{i} u\right\rangle=\|\operatorname{grad}(u)\|_{-1}^{2} .
$$

Consequently,

$$
P(\Omega)^{-1}=\operatorname{Inf}_{\substack{u \in M(\Omega) \\|u|_{2}=1}}(T u, u)_{2} .
$$

Important properties of this operator $T$ are as follows.

THEOREM 1

1) $T$ is a self-adjoint and coercive operator.

2) $P(\Omega)$ is the inverse of smallest spectral value of $T$.

3) $T-I$ is a harmonic mapping in $M(\Omega)$.

4) $\|T\|=1,1$ is a eigenvalue of $T$ and his eigenspace is infinite dimensional. 


\section{Maurice Gaultier and Mikel Lezaun}

\section{Proof}

(1) For all $(u, v) \in M(\Omega) \times M(\Omega)$ we have:

$$
\begin{gathered}
(T u, v)_{2}=\sum_{i=1}^{N}\left\langle\partial_{i} v,(-\Delta)^{-1} \circ \partial_{i} u\right\rangle=((\operatorname{grad}(u), \operatorname{grad}(v)))_{-1}=(v, T v)_{2}, \\
(T u, u)_{2}=\|\operatorname{grad}(u)\|_{-1}^{2} \geq P(\Omega)^{-1}|u|_{2}^{2}
\end{gathered}
$$

Then $T$ is a self-adjoint and coercive operator and $P(\Omega)^{-1}$ is the best value of the coercivity constant.

(2) We denote by $\sigma(T)$ the spectrum of $T$. It follows from (1) [1] that the residual spectrum of $T$ is empty, $\sigma(T)$ is closed and it lies in the closed interval $[m, M]$ on the real axis, where

$$
\begin{gathered}
m=\operatorname{Inf}_{\lambda \in \sigma(T)} \lambda=\operatorname{Inf}_{\substack{u \in M(\Omega) \\
|u|_{2}=1}}(T u, u)_{2}=P(\Omega)^{-1}, \\
M=\operatorname{Sup}_{\lambda \in \sigma(T)} \lambda=\operatorname{Sup}_{\substack{u \in M(\Omega) \\
|u|_{2}=1}}(T u, u)_{2}=\|T\| .
\end{gathered}
$$

So, $P(\Omega)^{-1} \in \sigma(T)$ and it is the smallest spectral value of $T$.

(3) For all $u \in M(\Omega)$ we have, in the sens of distributions on $\Omega$ :

$$
\Delta \circ T(u)=-\sum_{i=1}^{N} \Delta\left(\partial_{i} \circ(-\Delta)^{-1} \circ \partial_{i}\right)(u)=\Delta u
$$

So, $T(u)-u$ is a harmonic distribution on $\Omega$, thus it is a harmonic function on $\Omega$, for all $u \in M(\Omega)$.

(4) Let $H(\Omega)$ be the closed subspace of $M(\Omega)$ of harmonic functions:

$$
H(\Omega)=\{u \in M(\Omega) \mid \Delta u=0\}
$$

and we denote by $H(\Omega)^{\perp}$ the orthogonal complement of $H(\Omega)$ in $M(\Omega)$. 
Let $v \in H(\Omega)^{\perp}$ be such that $v \neq 0$ and $w \in H(\Omega)$. It follows from (3) that

$$
(T(v)-v, w)_{2}=(v, T(w)-w)_{2}=0 .
$$

Then $T(v)-v \in H(\Omega)^{\perp} \cap H(\Omega)$, consequently $T v=v$ and 1 is an eigenvalue of $T$.

Now let $\phi \in D(\Omega)$ be with $\Delta \phi \neq 0$, we have that $T(\Delta \phi)=\Delta \phi$. Then, the eigenspace corresponding to the eigenvalue 1 is infinite dimensional.

On the other hand, for all $v \in H(\Omega)^{\perp}, v \neq 0$, we have:

$$
|v|_{2}^{2}=(T(v), v)_{2} \leq\|T\||v|_{2}^{2}, \quad \text { from where }\|T\| \geq 1 .
$$

Moreover:

$$
\|T\|=\operatorname{Sup}_{\substack{u \in M(\Omega) \\ u \neq 0}} \frac{(T(u), u)_{2}}{|u|_{2}^{2}}=\operatorname{Sup}_{\substack{u \in M(\Omega) \\ u \neq 0}} \frac{\|\operatorname{grad}(u)\|_{-1}^{2}}{|u|_{2}^{2}} \leq 1 .
$$

Consequently, $\|T\|=1$. Finally $[1]$,

$$
\|T\|=\operatorname{Sup}_{\lambda \in \sigma(T)} \lambda .
$$

So, the eigenvalue 1 is the largest spectral value of $T$.

CoRollary 3.- If $u$ is an eigenvector of $T$ corresponding to an eigenvalue $\lambda \neq 1$, then $u$ is a harmonic function on $\Omega$.

In the following section, we are going to give a method to approximate the constant $P(\Omega)$.

5. Approximation of the Poincaré type constant $P(\Omega)$

$M(\Omega)$ is separable. Let $\left\{\varepsilon_{j} \mid 0<j<\infty\right\}$ be an orthonormal basis of $M(\Omega)$, we consider the sequence $\left\{M_{K}(\Omega)\right\}_{K>0}$ of finite dimensional subspaces of $M(\Omega)$ defined as follows: for all integer $K>0, M_{K}(\Omega)$ is spanned by the family of vectors $\left\{\varepsilon_{j} \mid 0<j \leq K\right\}$.

Then $M_{K}(\Omega) \subset M_{K+1}(\Omega), \forall K>0$, and for all $u \in M(\Omega)$, there exist a sequence $\left\{u_{K}\right\}_{K>0}, u_{K} \in M_{K}(\Omega)$, such that $\lim _{K \rightarrow \infty}\left|u-u_{K}\right|_{2}=0$. 
For all integer $K>0$, let us put

$$
\alpha_{K}=\operatorname{Inf}_{\substack{w \in M_{K}(\Omega) \\ w \neq 0}} \frac{\|\operatorname{grad}(w)\|_{-1}^{2}}{|w|_{2}^{2}}=\operatorname{Inf}_{\substack{w \in M_{K}(\Omega) \\ w \neq 0}} \frac{(T(w), w)_{2}}{|w|_{2}^{2}} .
$$

Then $\alpha_{K} \geq P(\Omega)^{-1}$ and $\alpha_{K} \geq \alpha_{K+1}, \forall K>0$. Thus, the sequence $\left\{\alpha_{K}\right\}_{K>0}$ is convergent and its limit $\alpha$ is such that $\alpha \geq P(\Omega)^{-1}$. On the other hand, let $u$ be some element of $M(\Omega)$. Then, there exist a sequence $\left\{u_{K}\right\}_{K>0}$ with $u_{K} \in M_{K}(\Omega)$, such that if $K \rightarrow \infty, u_{K} \rightarrow u$ in $L^{2}(\Omega)$ and therefore $\operatorname{grad}\left(u_{K}\right) \rightarrow \operatorname{grad}(u)$ in $\left(H^{-1}(\Omega)\right)^{N}$. Furthermore, we have $\left\|\operatorname{grad}\left(u_{K}\right)\right\|_{-1}^{2} \geq \alpha_{K}\left|u_{K}\right|_{2}^{2}$. Passing to limit when $K \rightarrow \infty$, we obtain $\|\operatorname{grad}(u)\|_{-1}^{2} \geq \alpha|u|_{2}^{2}$. This result implies that $\alpha \leq P(\Omega)^{-1}$ and consequently $P(\Omega)^{-1}=\alpha$.

Now, we are going to specify the elements of sequence $\left\{\alpha_{K}\right\}_{K>0}$.

Let $K$ be some positive integer. Each $u \in M_{K}(\Omega)$ has a unique decomposition $u=\sum_{j=1}^{K} \lambda_{j} \varepsilon_{j}$. Put $\chi=G u=\left(\lambda_{1}, \lambda_{2}, \ldots, \lambda_{K}\right) \in \mathbb{R}^{K}$.

In what follows, $M_{K}(\Omega)$ is equipped with the norm induced by $L^{2}(\Omega)$ and $\mathbb{R}^{K}$ is equipped with the usual euclidean norm $\|\cdot\|_{0}$. Then $G$ is an isometric isomorphism from $M_{K}(\Omega)$ onto $\mathbb{R}^{K}$.

On the other hand,

$$
(T u, u)_{2}=\left(T \circ G^{-1} \chi, G^{-1} \chi\right)_{2} \geq P(\Omega)^{-1}\|\chi\|_{0}^{2} .
$$

Therefore

$$
\chi \rightarrow Q_{K}(\chi)=\left(T \circ G^{-1} \chi, G^{-1} \chi\right)_{2}=\sum_{i, j=1}^{K} \lambda_{i} \lambda_{j}\left(T \varepsilon_{i}, \varepsilon_{j}\right)_{2}
$$

is a positive definite quadratic form on $\mathbb{R}^{K}$ and furthermore:

$$
\alpha_{K}=\operatorname{Inf}_{\substack{\chi \in \mathbb{R}^{K} \\ \chi \neq 0}} \frac{Q_{K}(\chi)}{\|\chi\|_{0}^{2}} .
$$

So, we have proved the following result.

Proposition 2.- $\alpha_{K}$ is the smallest eigenvalue (minimum of a Rayleigh quotient) of the matrix $A_{K}$ of the quadratic form defined on $\mathbb{R}^{K}$ by formula (6) and $\alpha_{K} \rightarrow P(\Omega)^{-1}$, when $K \rightarrow \infty$. 


\section{Two particular cases}

6.1 The open is the rectangle $\Omega=\left\{(x, y) \in \mathbb{R}^{2} \mid 0<x<L, 0<y<\ell\right\}$

We use the previous results with $N=2$.

We must now calculate the elements $\left(T \varepsilon_{i}, \varepsilon_{j}\right)_{2}$ of the matrix $A_{K}$. For all $(m, p) \in \mathbb{N}^{2}$, we put

$$
\omega_{m}=\frac{m \pi}{L}, \quad \delta_{p}=\frac{p \pi}{\ell}, \quad \alpha_{m, p}=\omega_{m}^{2}+\delta_{p}^{2}
$$

and introduce real positive numbers $c_{m, p}$ defined by

$$
c_{0, p}^{2}=c_{m, 0}^{2}=\frac{1}{2} c_{m, p}^{2}=\frac{2}{L \ell}, \quad \text { for } m \geq 1 \text { and } p \geq 1 .
$$

We choose the following orthonormal basis of $M(\Omega)$ :

$$
\begin{array}{r}
\left\{e_{m, p}(x, y)=c_{m, p} \cos \left(\omega_{m} x\right) \cos \left(\delta_{p} y\right) \mid(x, y) \in \Omega,(m, p) \in \mathbb{N}^{2},\right. \\
m+p \geq 1\} .
\end{array}
$$

The elements of the matrix $A_{K}$ are the following real numbers:

$$
\begin{aligned}
\left(T e_{m, p}, e_{j, q}\right)_{2} \quad \text { with }(m, p) \in \mathbb{N}^{2},(j, q) \in \mathbb{N}^{2} & \\
& 0 \leq m, p, j, q \leq K, m+p \geq 1 \text { and } j+q \geq 1 .
\end{aligned}
$$

More precisely, $\left(T e_{m, p}, e_{j, q}\right)_{2}$ is the element of the $(m(K+1)+p)$-th row and $(j(K+1)+q)$-th columm of the matrix $A_{K}$.

In order to give explicit values of $\left(T e_{m, p}, e_{j, q}\right)_{2}$, it is convenient to introduce $a(r, s)$ for $(r, s) \in \mathbb{N}^{2}$ with $r \geq 1$ and $s \geq 1$, given by

$$
a(r, 0)=a(0, s)=1, \quad a(r, s)=\sqrt{2} .
$$

The calculation of the scalar products $\left(T e_{m, p}, e_{j, q}\right)_{2}$ is long but not very difficult. We obtain:

(i) $\left(T e_{m, p}, e_{j, q}\right)_{2}=0$ if $m \neq j$ and $p \neq q$;

(ii) $\left(T e_{m, p}, e_{m, q}\right)_{2}=-\frac{\sqrt{2} a(p, q) \omega_{m}^{3}\left(1+(-1)^{p+q}\right) B_{m, p}}{\ell \alpha_{m, p} \alpha_{m, q}}$ if $p \neq q$, 
(iii) $\left(T e_{m, p}, e_{j, p}\right)_{2}=-\frac{\sqrt{2} a(m, j) \delta_{p}^{3}\left(1+(-1)^{m+j}\right) \Lambda_{m, p}}{L \alpha_{m, p} \alpha_{j, p}}$ if $m \neq j$,

(iv) $\left(T e_{m, p}, e_{m, p}\right)_{2}=1-a(m, p)^{2}\left(\frac{2 \omega_{m}^{3} B_{m, p}}{\ell \alpha_{m, p}^{2}}-\frac{2 \delta_{p}^{3} \Lambda_{m, p}}{L \alpha_{m, p}^{2}}\right)$,

where

$$
\begin{gathered}
B_{0, p}=0, B_{m, p}=\frac{\exp \left(\omega_{m} \ell\right)+\exp \left(-\omega_{m} \ell\right)-2(-1)^{p}}{\exp \left(\omega_{m} \ell\right)-\exp \left(-\omega_{m} \ell\right)}, \forall m \geq 1, \forall p \geq 0 \\
\Lambda_{m, 0}=0, \Lambda_{m, p}=\frac{\exp \left(\delta_{p} L\right)+\exp \left(-\delta_{p} L\right)-2(-1)^{m}}{\exp \left(\delta_{p} L\right)-\exp \left(-\delta_{p} L\right)}, \forall m \geq 0, \forall p \geq 1 .
\end{gathered}
$$

Remark 1. $-A_{K}$ and consequently $P(\Omega)^{-1}$, depend only on the ratio of the dimensions of the rectangle.

Numerical results. - We have performed a few numerical tests. Let $K$ be a positive integer. We have computed an approximate value of the smallest eigenvalue $\alpha_{K}$ of the matrix $A_{K}$ by means of the power of Mises [2, pp. 226-227]. We stopped this calculatioin when the relative error was less than $10^{-9}$. We have ascertained that sequence $\left\{\alpha_{K}\right\}_{K>0}$ converges quickly.

The above mentioned values of the constant $P(\Omega)^{-1}$ have been rounded up to the 3 -th decimal place.

$$
\begin{array}{ll}
L=1, & \ell=1: P(\Omega)^{-1}=0.226 \\
L=2, & \ell=1: P(\Omega)^{-1}=0.151 \\
L=4, & \ell=1: P(\Omega)^{-1}=0.047 .
\end{array}
$$

6.2 Then open is $\Omega=\left\{\left(x_{1}, x_{2}, \ldots, x_{n+2}\right) \in \mathbb{R}^{n+2} \mid x_{1}^{2}+\cdots+x_{n+2}^{2}<1\right\}$ with $n \in \mathbb{N}$

In this case, we are able to give the spectrum of $T$. In that follows, the following proposition is essential.

Proposition 3.- Each harmonic homogeneous polynomial in $\Omega$ of degree $m \geq 1$ is an eigenvector of $T$ corresponding to the eigenvalue $m /(2 m+n)$. 
Proof. - Let $u\left(x_{1}, x_{2}, \ldots, x_{n+2}\right)$ be a harmonic homogeneous polynomial in $\Omega$ of degree $m \geq 1$. We have

$$
\sum_{i=1}^{n+2} x_{i} \partial_{i} u=m u \text {. }
$$

Hence, for all $j=1,2, \ldots, n+2$,

$$
\partial_{j} u+\sum_{i=1}^{n+2} x_{i} \frac{\partial^{2} u}{\partial x_{j} \partial x_{i}}=m \partial_{j} u .
$$

On the other hand:

$$
\begin{aligned}
\Delta\left(\left(x_{1}^{2}+x_{2}^{2}+\cdots+x_{n+2}^{2}-1\right) \partial_{j} u\right)= & 2(n+2) \partial_{j} u+4 \sum_{i=1}^{n+2} x_{1} \frac{\partial^{2} u}{\partial x_{i} \partial x_{j}}+ \\
& \left.+x_{1}^{2}+x_{2}^{2}+\cdots+x_{n+2}^{2}-1\right) \partial_{j}(\Delta u),
\end{aligned}
$$

and from (7)

$$
\begin{aligned}
\Delta\left(\left(x_{1}^{2}+x_{2}^{2}+\cdots+x_{n+2}^{2}-1\right) \partial_{j} u\right) & =2(n+2) \partial_{j} u+4(m-1) \partial_{j} u \\
& =(2 n+4 m) \partial_{j} u
\end{aligned}
$$

Since the function $\left(x_{1}^{2}+x_{2}^{2}+\cdots+x_{n+2}^{2}-1\right) \partial_{j} u \in D(\Omega)$, it follows from (8) that

$$
(-\Delta)^{-1}\left(\partial_{j} u\right)=-\frac{1}{4 m+2 n}\left(x_{1}^{2}+x_{2}^{2}+\cdots+x_{n+2}^{2}-1\right) \partial_{j} u .
$$

Therefore,

$$
\begin{aligned}
& -\operatorname{div} \circ(-\Delta)^{-1} \circ \operatorname{grad}(u)= \\
& =\frac{1}{4 m+2 n}\left(2 \sum_{j=1}^{n+2} x_{j} \partial_{j} u+\left(x_{1}^{2}+x_{2}^{2}+\cdots+x_{n+2}^{2}-1\right) \Delta u\right), \\
& T u=\frac{1}{2 m+n} \sum_{j=1}^{n+2} x_{j} \partial_{j} u=\frac{m}{2 m+n} u .
\end{aligned}
$$

We denote by $H_{m}(\Omega)$ the eigenspace of $T$ corresponding to the eigenvalue $m /(2 m+n)$ with $m \in \mathbb{N}^{*}$.

We recall that $H(\Omega)$ is the subspace of $M(\Omega)$ of harmonic functions and $H(\Omega)^{\perp}$ its orthogonal complement in $M(\Omega)$. We have the following result. 
Proposition 4 [3]. - The family of harmonic homogeneous polynomials in $\Omega$ of degree $m \geq 1$ is free and total in $H(\Omega)$.

Proposition 5.- The orthogonal complement $H(\Omega)^{\perp}$ of $H(\Omega)$ in $M(\Omega)$ is the eigenspace of $T$ corresponding to the eigenvalue 1 .

Proof. - We denote by $H_{-n}(\Omega)$ the eigenspace of $T$ corresponding to the eigenvalue 1. It is proved in theorem 1(4) that $H(\Omega)^{\perp} \subset H_{-n}(\Omega)$.

On the other hand, eigenvectors corresponding to different eigenvalues of $T$ are orthogonal; from propositions 3 and 4 we have $H_{-n}(\Omega) \subset H(\Omega)^{\perp}$.

Then, $H_{-n}(\Omega)=H(\Omega)^{\perp}$, and consequently,

$$
M(\Omega)=H_{-n}(\Omega) \oplus H(\Omega) .
$$

COROLlaRY 4. - The only eigenvalues of $T$ are 1 and $m /(2 m+n)$ with $m \in \mathbb{N}^{*}$.

$T$ is a bounded self-adjoint operator on $M(\Omega)$. Its spectrum $\sigma(T)$ is partitioned into two disjoint sets: the point spectrum $\sigma_{p}(T)$ and the continuous spectrum $\sigma_{c}(T)$.

Now, we are going to specify two cases.

1) $n=0, \Omega=\left\{\left(x_{1}, x_{2}\right) \in \mathbb{R}^{2} \mid x_{1}^{2}+x_{2}^{2}<1\right\}$

In this case, the harmonic homogeneous polynomials of degree $m \geq 1$ are eigenvectors of $T$ corresponding to the eigenvalue $1 / 2$.

Theorem 3. - T has a pure point spectrum. $1 / 2$ and 1 are the only eigenvalues of $T$.

Proof. - It follows from corollary 4 that $\sigma_{p}(T)=\{1 / 2,1\}$.

Now, let $\alpha \in \mathbb{R}$ be different of $1 / 2$ or 1 , and $v \in M(\Omega)$. We have

$$
v=v_{1 / 2}+v_{0} \quad \text { with } v_{1 / 2} \in H_{1 / 2}(\Omega) \text { and } v_{0} \in H(\Omega)^{\perp},
$$

where $H_{1 / 2}(\Omega)$ is the eigenspace of $T$ corresponding to the eigenvalue $1 / 2$. We take

$$
w=\frac{2}{1-2 \alpha} v_{1 / 2}+\frac{2}{1-\alpha} v_{0} ;
$$

then $w \in M(\Omega)$, and we check that $(T-\alpha I) w=v$. Therefore, $\alpha \notin \sigma(T)$. 
Remark 2. - The two eigenspaces of $T$ are infinite dimensional.

2) $n \geq 1, \Omega=\left\{\left(x_{1}, \ldots, x_{n+2}\right) \in \mathbb{R}^{n+2} \mid x_{1}^{2}+\cdots+x_{n+2}^{2}<1\right\}$

THEOREM 2.- $\sigma_{p}(T)=\left\{1, m /(2 m+n) \mid m \in \mathbb{N}^{*}\right\}$ and $\sigma_{c}(T)=$ $\{1 / 2\}$.

Proof. - From corollary 4,

$$
\sigma_{p}(T)=\left\{1, \frac{m}{2 m+n} \mid m \in \mathbb{N}^{*}\right\} .
$$

Now, let $\alpha \in \mathbb{R}$ be different of $1 / 2,1$ or $m /(2 m+n)$ with $m \in \mathbb{N}^{*}$, and $v \in M(\Omega)$. We have

$$
v=\sum_{m=1}^{\infty} v_{m}+v_{0} \quad \text { with } v_{m} \in H_{m}(\Omega) \text { and } v_{0} \in H(\Omega)^{\perp} .
$$

We take

$$
w=\sum_{m=1}^{\infty} \frac{2 m+n}{m-\alpha(2 m+n)} v_{m}+\frac{1}{1-\alpha} v_{0} ;
$$

then $w \in M(\Omega)$, and we check that $(T-\alpha I) w=v$. Therefore, $\alpha \notin \sigma(T)$.

Consequently, the limit $1 / 2$ of the eigenvalues is the only element of the continuous spectrum of the operator $T$.

COROLlaRY 5.- The eigenspace of $T$ corresponding to the eigenvalue $m /(2 m+n)$ is finite dimensional.

Proof. - Let $P_{m}$ be the vector subspace of $M(\Omega)$ spanned by the harmonic homogeneous polynomials of degree $m \geq 1\left(\operatorname{dim} P_{m}<+\infty\right)$. It follows from propositions 3 and 4 that $H(\Omega)$ is spanned by the family $\left\{P_{m} \mid m \geq 1\right\}$ :

$$
M(\Omega)=\bigotimes_{m \geq 1} P_{m} \oplus H(\Omega)^{\perp}
$$

On the other hand, suppose that there is an eigenvector $u$ corresponding to the eigenvalue $\lambda_{m}=m /(2 m+n)$, such that $u \notin P_{m}$. From corollary 3 , $u \in H(\Omega)$. Since $u \perp H(\Omega)^{\perp}, u$ can be written

$$
u=\sum_{\substack{j \geq 1 \\ j \neq m}} u_{j}, \quad \text { where } u_{j} \in P_{j} .
$$


Then

$$
\lambda_{m} u=T u=\sum_{\substack{j \geq 1 \\ j \neq m}} \lambda_{j} u_{j}, \quad \text { with } \lambda_{j}=\frac{j}{2 j+n}
$$

and

$$
\sum_{\substack{j \geq 1 \\ j \neq m}}\left(\lambda_{j}-\lambda_{m}\right) u_{j}=0
$$

But this is impossible because the family $\left\{u_{j}\right\}_{j \geq 1}$ is free.

Hence $u \in P_{m}$; thus $P_{m}$ is the eigenspace of $T$ corresponding to the eigenvalue $m /(2 m+n)$ and the corollary is proved.

We recall that the eigenspace of $T$ corresponding to 1 is infinite dimensional.

Corollary $6 .-$ If $\Omega$ is the ball $\Omega=\left\{\left(x_{1}, \ldots, x_{N}\right) \mid x_{1}^{2}, \ldots, x_{N}^{2}<1\right\}$ with $N \geq 2$, then $P(\Omega)=N$.

Remark 3. - The spectrum of the operator $T$ is independent of the radius of the ball in $\mathbb{R}^{N}$.

Remark 4. - Now we are going to give a family of eigenvectors of $T$ which is total in $M(\Omega)$.

First we consider the orthogonal basis for $M(\Omega)$ formed by the eigenvectors of $-\Delta$ (in $\left.L^{2}(\Omega)\right)$ for the Neumann problem.

To write these functions, the appropriate polar co-ordinates are

$$
\begin{aligned}
& x_{1}=\rho \cos \theta_{1}, \\
& x_{2}=\rho \sin \theta_{1} \cos \theta_{2}, \\
& x_{3}=\rho \sin \theta_{1} \sin \theta_{2} \cos \theta_{3}, \\
& \ldots \\
& x_{n+1}=\rho \sin \theta_{1} \sin \theta_{2} \cdots \sin \theta_{n} \cos \varphi, \\
& x_{n+2}=\rho \sin \theta_{1} \sin \theta_{2} \cdots \sin \theta_{n} \sin \varphi ;
\end{aligned}
$$

with $0 \leq \varphi \leq 2 \pi, 0 \leq \theta_{1}, \theta_{2}, \ldots, \theta_{n} \leq \pi$ and $0 \leq \rho<1$. 
For simplicity, we put:

$$
L_{\nu_{i}+(n-i) / 2}^{\nu_{i+1}+(n-i) / 2}(t)=P_{\nu_{i}+(n-i) / 2}^{\nu_{i+1}+(n-i) / 2}(t), \quad t \in[0,1]
$$

if $n-i$ is a positive even integer and

$$
L_{\nu_{i}+(n-i) / 2}^{\nu_{i+1}+(n-i) / 2}(t)=Q_{\nu_{i}+(n-i) / 2}^{\nu_{i}+1+(n-i) / 2}(t), \quad t \in[0,1]
$$

if $n-i$ is a positive odd integer, where $P_{\mu}^{\eta}$ is the associate Legendre function of the first kind of order $\mu$ and degree $\eta$, and $Q_{\mu}^{\eta}$ is the associate Legendre function of the second kind of order $\mu$ and degree $\eta$ [8].

In these polar co-ordinates this orthogonal basis for $M(\Omega)$ is the family of functions $\left\{\Phi_{\nu, k}^{q} \mid k \in \mathbb{N}\right\} \cup\left\{\Psi_{\nu, k}^{q} \mid k \in \mathbb{N}^{*}\right\}$ defined by

$$
\begin{aligned}
\Phi_{\nu, k}^{q}= & \rho^{-n / 2} J_{\nu_{1}+n / 2}\left(\lambda_{\nu_{1}+n / 2, q} \rho\right) \times \\
& \times\left(\prod_{i=1}^{n}\left(L_{\nu_{i}+(n-i) / 2}^{\nu_{i+1}+(n-i) / 2}\left(\cos \theta_{i}\right)\left(\operatorname{cosec} \theta_{i}\right)^{(n-i) / 2}\right)\right) \cos (k \varphi) \\
\Psi_{\nu, k}^{q}= & \rho^{-n / 2} J_{\nu_{1}+n / 2}\left(\lambda_{\nu_{1}+n / 2, q} \rho\right) \times \\
& \times\left(\prod_{i=1}^{n}\left(L_{\nu_{i}+(n-i) / 2}^{\nu_{i+1}+(n-i) / 2}\left(\cos \theta_{i}\right)\left(\operatorname{cosec} \theta_{i}\right)^{(n-i) / 2}\right)\right) \sin (k \varphi) .
\end{aligned}
$$

with $q \in \mathbb{N}^{*}, \nu=\left(\nu_{1}, \nu_{2}, \ldots, \nu_{n}\right) \in \mathbb{N}^{n}, k=\nu_{n+1} \leq \nu_{n} \leq \cdots \leq \nu_{1}$; where $J_{\nu_{1}+n / 2}$ is the Bessel function of the first kind of order $\nu_{1}+n / 2$ [9], and $\lambda_{\nu_{1}+n / 2, q}$ are the positive roots of equation

$$
\left.\frac{\mathrm{d}}{\mathrm{d} \rho}\left(\rho^{-n / 2} J_{\nu_{1}+n / 2}(\lambda \rho)\right)\right|_{\rho=1}=0 .
$$

If we write the harmonic homogeneous polynomials of degree $\nu_{1} \geq 1$ in these polar co-ordinates, we have

$$
\begin{aligned}
& u_{\nu, k}^{(1)}\left(\rho, \theta_{1}, \ldots, \theta_{n}, \varphi\right)= \\
& \quad=\rho^{\nu_{1}}\left(\prod_{i=1}^{n}\left(L_{\nu_{i}+(n-i) / 2}^{\nu_{i+1}+(n-i) / 2}\left(\cos \theta_{i}\right)\left(\operatorname{cosec} \theta_{i}\right)^{(n-i) / 2}\right)\right) \cos (k \varphi), \quad k \in \mathbb{N},
\end{aligned}
$$




$$
\begin{aligned}
& u_{\nu, k}^{(2)}\left(\rho, \theta_{1}, \ldots, \theta_{n}, \varphi\right)= \\
& \quad=\rho^{\nu_{1}}\left(\prod_{i=1}^{n}\left(L_{\nu_{i}+(n-i) / 2}^{\nu_{i+1}+(n-i) / 2}\left(\cos \theta_{i}\right)\left(\operatorname{cosec} \theta_{i}\right)^{(n-i) / 2}\right)\right) \sin (k \varphi), \quad k \in \mathbb{N}^{*}
\end{aligned}
$$

where $\nu=\left(\nu_{1}, \nu_{2}, \ldots, \nu_{n}\right) \in \mathbb{N}^{n}, k=\nu_{n+1} \leq \nu_{n} \leq \cdots \leq \nu_{1}$. We say that this family of harmonic homogeneous polynomials is an orthogonal basis for $H(\Omega)$ and, as proved in proposition 3, they are eigenvectors of $T$ corresponding to the eigenvalue $\nu_{1} /\left(2 \nu_{1}+n\right)$.

Now we consider the functions $\Phi_{0,0}^{q}, \Phi_{\nu, k}^{q}-\gamma_{\nu, k}^{q} u_{\nu, k}^{(1)}$ and $\Psi_{\nu, k}^{q}-\delta_{\nu, k}^{q} u_{\nu, k}^{(2)}$, with $q \in \mathbb{N}^{*}, k \in \mathbb{N}^{*}, \nu=\left(\nu_{1}, \nu_{2}, \ldots, \nu_{n}\right) \in \mathbb{N}^{n}, k=\nu_{n+1} \leq \nu_{n} \leq \cdots \leq \nu_{1}$; where

$$
\gamma_{\nu, k}^{q}=\frac{\left(\Phi_{\nu, k}^{q}, u_{\nu, k}^{(1)}\right)_{2}}{\left|u_{\nu, k}^{(1)}\right|_{2}^{2}} \quad \text { and } \quad \delta_{\nu, k}^{q}=\frac{\left(\Psi_{\nu, k}^{q}, u_{\nu, k}^{(2)}\right)_{2}}{\left|u_{\nu, k}^{(2)}\right|_{2}^{2}}
$$

We obtain that these functions are eigenvectors of $T$ corresponding to the eigenvalue 1 .

Finally, we prove that the family of eigenvectors of $T$

$$
\left\{u_{\nu, k}^{(1)}, u_{\nu, k}^{(2)}, \Phi_{0,0}^{q}, \Phi_{\nu, k}^{q}-\gamma_{\nu, k}^{q} u_{\nu, k}^{(1)}, \Psi_{\nu, k}^{q}-\delta_{\nu, k}^{q} u_{\nu, k}^{(2)}\right\}
$$

with $q \in \mathbb{N}^{*}, k \in \mathbb{N}^{*}, \nu=\left(\nu_{1}, \nu_{2}, \ldots, \nu_{n}\right) \in \mathbb{N}^{n}, k=\nu_{n+1} \leq \nu_{n} \leq \cdots \leq \nu_{1}$ is total in $M(\Omega)$.

\section{Acknowledgments}

A part of this work was done during a visit of the first author to the Departement of Applied Mathematics and Statitics of the University of Bilbao (Universidad del País Vasco).

The authors express their thanks to E. Sainz de la Maza for his advice concerning the numerical part of this paper. Thanks also extend to J. Duoandikoetxea for his important suggestions. 
Spectral stydy of a self-adjoint operator on $L^{2}(\Omega)$

\section{References}

[1] BrÉzIs (H.) .- Analyse fonctionnelle, Théorie et Applications. Masson, Paris (1983).

[2] Carnhan (B.), Luther (H. A.) and Wilker (J. O.) - - Applied Numerical Methods, John Wiley \& Sons (1969).

[3] Dautrey (R.) and Lions (J.-L.) .- Analyse Mathématiques et Calcul Numérique pour les Sciences et les Techniques, Masson, Paris (1984).

[4] Gaultier (M.) and Lezaun (M.) .- An existence and uniqueness theorem for the transfer of mass and heat in a rectangular cavity, I.M.A. J. Appl. Math. 48 (1992), pp. 125-148.

[5] Grisvard (P.) .- Elliptic Problems in Nonsmooth Domains, Pitman, London (1985).

[6] NECAS (J.) .- Les méthodes directes en théories des équations elliptiques, Masson, Paris (1967).

[7] Teman (R.) .- Navier-Stokes Equations, North-Holland, Amsterdam (1977).

[8] WANG (Z. X.) and GUO (D. R.) . - Special functions, World Scientific Publishing Co., Singapore (1989).

[9] Watson (G. N.) .- A treatise on the theory of Bessel functions, 2nd ed., Cambridge University Press, London (1944). 